\title{
ComSens: Exploiting Pilot Diversity for Pervasive Integration of Communication and Sensing in MIMO-TDD-Frameworks
}

\author{
Mohammadreza Mousaei, Mojtaba Soltanalian, and Besma Smida, Senior Member, IEEE \\ Department of Electrical and Computer Engineering, University of Illinois at Chicago (UIC), Chicago, IL, USA \\ Email: \{mmousa3, msol, smida\} @uic.edu
}

\begin{abstract}
In this paper, we propose a fully-integrated radar and communication system - named ComSens. We utilize two different pilot sequences (one for uplink and one for downlink) with the condition that they must be uncorrelated to each other. Within such a framework, the signal received from end-user and the backscattered signal from the desired objects have uncorrelated pilots. Thus, the base-station is able to distinguish data signal from user and back-scattered signal from object. We assume a time division duplex (TDD) framework. The pilot sequences are designed for MIMO channels. We evaluate channel MSE as a figure of merit for communication system. We also show that the designed pilots are uncorrelated for a range of time lags. Moreover, designed uplink pilot has negligable autocorrelation for a range of time lags leading to an impulse-like autocorrelation for radar sensing.
\end{abstract}

\section{INTRODUCTION}

Due to the increasing demand in wireless communication services, achieving higher data rates and more reliable transmissions have become a fundamental goal [1]-[4]. Given the ever-increasing demand for both high-speed data services and accurate remote sensing capabilities, modern wireless systems will increasingly require more efficient strategies for use of the available frequency spectrum [5]-[9]. In particular, the coexistence of communication and radar systems has recently attracted a significant research interest [10]-[14]. For example, different schemes for coexisting communication and radar systems has been proposed; see e.g. [15]-[21] and the references therein. While integrating radar and communication operation in one system has been considered in the literature, such efforts are typically centered around incorporating communication as a secondary operation alongside a primary radar operation. The research in [15] exploits the main lobe of the beam for radar purposes, and the sidelobes (which are of no significance to the radar pulse compression) for data transmission purposes. The research works [16], [17] approach the same problem by devising similar methods to allow comparably low data rates into an already existing radar system.

In this work, we propose an integrated system of communication and sensing (which we call ComSens) that relies on the communication pilot overhead- thus paving the way for pilot design and exploiting pilot diversity to achieve a satsifactory performance in both communication and radar tasks. Note that:

Pilot (or training) based channel estimation is very common [22], [23]. Accurate knowledge of channel state information (CSI) is important for wireless communication systems [24], [25]. Most modern wireless systems acquire the CSI with the assistance of pilot signals (a.k.a. training sequences) that are inserted within the transmit signals periodically [26], [27]. In such scenarios, the transmitter sends training sequences - known to the receiver - enabling the receiver to perform channel estimation on the basis of the received training symbols.

Communication devices are more ubiquitous than radar systems [28], [29]. We note that incorporating the communication signals in the primary radar probing waveforms may not be an efficient fusion of communication and radar systems. In fact, the communication task must play a primary rule not only because of the pervasive usage of comuunication devices, but also the fact that the communication systems typically require a larger capacity of conveying information than radar systems. Additionally, considering the communication operation as the primary lays the ground for making the radar systems ubiquitous (for example having radar capability on cellphones).

\section{A. Contributions}

The key departure from prior works on integrated radarcommunication systems is that we (a) incorporate a radar system in an already existing communication system, particularly by (b) using the novel idea of designing different training signals such that sensing and communication can co-exist. (c) incorporating such a system in TTD framework in a MIMO system and designing the pilot sequences.

\section{B. Notation}

We use bold lowercase letters for vectors/sequences and bold uppercase letters for matrices. $(\cdot)^{T},(\cdot)^{*}$ and $(\cdot)^{H}$ denote the vector/matrix transpose, the complex conjugate, and the Hermitian transpose, respectively. $\|x\|_{n}$ or the $l_{n}$-norm of the vector $\boldsymbol{x}$ is defined as $\left(\sum_{k}|\boldsymbol{x}(k)|^{n}\right)^{\frac{1}{n}}$ where $\{\boldsymbol{x}(k)\}$ are the entries of $\boldsymbol{x}$. The Frobenius norm of a matrix $\boldsymbol{X}$ (denoted by $\|\boldsymbol{X}\|_{F}$ ) with entries $\{\boldsymbol{X}(k, l)\}$ is equal to $\left(\sum_{k, l}|\boldsymbol{X}(k, l)|^{2}\right)^{\frac{1}{2}}$. Finally, $\mathbb{R}$ and $\mathbb{C}$ represent the set of real and complex numbers, respectively.

\section{FUSION OF COMMUNICATION AND RADAR OPERATIONS}

In this section, we describe the problem settings and explain our proposed scheme in more details.

\section{A. The Proposed Integration Scheme}

The ComSens framework operates by exploiting the two-way communication between the base-station and end-users. Before 


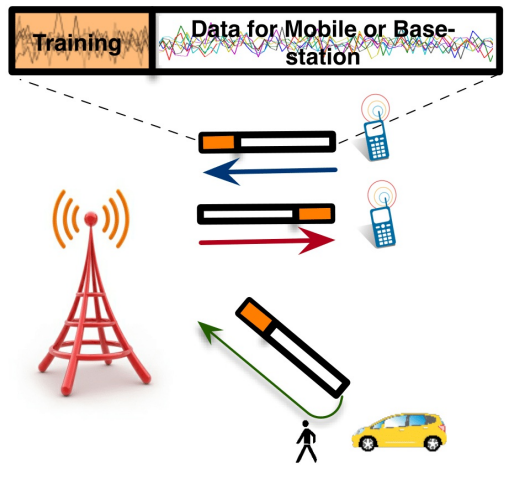

Base-Station

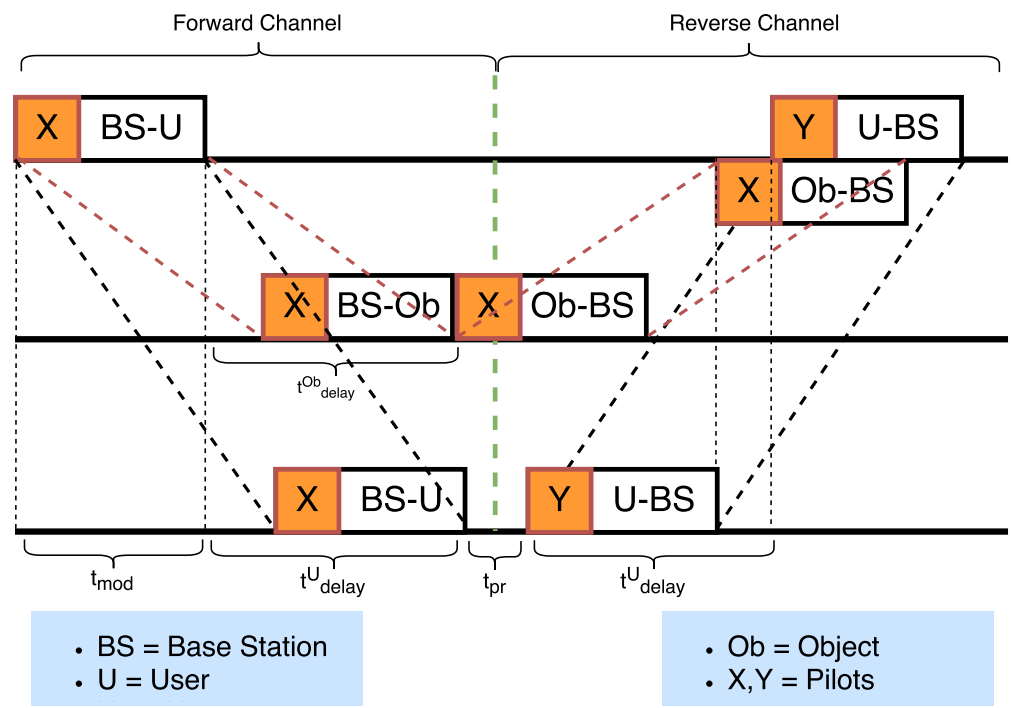

Fig. 1. The ComSens communication and sensing integration framework.

discussing the issue of designing the pilot sequences, we will first address how the base-station and end-user exchange messages and sense the environment at the same time and over the same frequency. We consider a model with $M$-user multiple-accessbroadcast channel (MABC) as depicted in Fig. 1. Note that such a system model, in which several end-users wish to exchange messages with a central node, or base-station, is a model that captures the behavior of current and future cellular networks. We assume half-duplex end-user nodes that may transmit or receive at a given time, on a given frequency, but not both, leading to the need to describe protocols, or which nodes transmit when. We consider time division duplex (TDD) two-way system as duplex scheme and for multiple access both FDMA and TDMA can be used. For each user, time is devided into forward channel and reverse channel (as in TDD scheme). During the former time, Base-station transmits the packet and during the latter user transmits the packet. The base-station (BS) sends a packet $s$ to the end-user $U$. The end-user can extract its own message after channel estimation using the downlink pilot - labeled $\boldsymbol{X}$. Contrary to most of the current works on integrated radar and communication systems, the data transmission proposed here is similar to the conventional half-duplex transmission. This guarantees a high-data rate to efficiently accommodate downlink traffic. At the same time, the packet $s$ is reflected from objects in the neighborhood. The base-station observes the echo of its own transmit signal, and detects the presence of objects and their distance and relative velocity. With ComSens, the base-station jointly estimates the radar return and extract the uplink message from end-user $\mathrm{U}$ after channel estimation using uplink pilot labeled $\boldsymbol{Y}$. The principal constraint in the performance of radar sensing is the simultaneous reception of the radar echo and uplink packet. Therefore the main goal of this work is to design the uplink and downlink pilot sequences. We design the two pilot sequences to be uncorrelated to each other so that they can be distinguished from each other at the base-station. After separation of two pilots, the base-station uses the packet with uplink pilot for communication purposes and the reflected downlink packet for sensing.

Remark: From the above discussion it must be clear that ComSens uplink communications may be subject to interference from the radar echo. Note that the echo signal is received at the base-station with high attenuation due to the two-way link (from the base-station to the object and from the object to the base-station) and the absorption at the object so its impact on the uplink communication is negligible.

\section{B. Time and Range Analysis}

\section{Limitations:}

Consider one TDD frame for an end-user $\mathrm{U}$ (as it is shown in Fig. 1). At the forward channel time, base-station transmits the packet. End-user receives the packet at $t_{\text {mod }}+t_{\text {delay }}^{U}$ where $t_{m o d}$ is the modulation and transmission time and $t^{U}$ delay is the propagation time between end-user and the base-station. Packet is processed at the end-user in $t_{p r}$ time. Then, end-user transmits the packet in the reverse channel time and base-station receives it at the time $t_{1}=t_{\text {mod }}+2 t_{\text {delay }}^{U}+t_{p r}$. On the other hand, transmitted packet from the base-station is also received at the object at the time $t_{m o d}+t_{\text {delay }}^{O b}$ where $t_{\text {delay }}^{O b}$ is the propagation time between object and the base-station. The packet is then back-scattered from the object and received at the base-station at the time $t_{2}=t_{\text {mod }}+2 t_{\text {delay }}^{\text {Ob }}$. We design downlink pilot and uplink pilot to be uncorrelated to each other for $k$ time lags. Therefore, if two received signals (from user and object) have arrival time difference $\left(t_{2}-t_{1}\right)$ of at most $k$, they are distinguishable from each other. On the other hand, if $t_{2}-t_{1}>k$, the radar signal cannot be recognized and it will be considered as weak interference for communication system. Consequently, our proposed integrated radar system will perform when $t_{2}-t_{1} \leq k$. Substituting $t_{1}$ and $t_{2}$ we have:

$$
t_{\text {delay }}^{U}-t_{\text {delay }}^{O b} \leq \frac{t_{p r}+k}{2}
$$


where

$$
t_{\text {delay }}^{O b, U}=\frac{d^{O b, U}}{\nu T_{s}}
$$

and $d^{U}$ and $d^{O b}$ are respectively the distance of user and the object from the base-station, $T_{s}$ is symbol time in our system and $\nu$ is the speed of electromagnetic wave in the space. Using Eq. (1, 2) we have

$$
d^{O b} \leq d^{U}+\frac{\nu T\left(t_{p r}+k\right)}{2}
$$

2. Practical Scenario: Communication cell towers have a range between $35 \mathrm{~km}$ to $72 \mathrm{~km}$. We consider our user to be (as a medium distance) at the distance $d^{U}=25 \mathrm{~km}$ of the base-station. Assume that the symbol time $T_{s}=25 \mu \mathrm{s}$ and processing time $t_{p r}=T_{s}$ where speed of electromagnetic wave is $\nu=3 \times 10^{8}$, assuming we design our pilots to be uncorrelated for $k=4$. Such a system would have a radar range of $43.75 \mathrm{~km}$ $\left(d^{O b} \leq 43.75 \mathrm{~km}\right)$.

\section{Channel Model}

We consider the same settings as in [30] and [31]. More precisely, we consider a narrowband block fading point-to-point MIMO channel with $n_{T}$ transmit and $n_{R}$ receive antennas. Assume that $\boldsymbol{P} \in \mathbb{C}^{B \times n_{T}}$ be a matrix whose rows are the pilot sequence at each transmitter antenna. At the training phase, channel can be described as

$$
\boldsymbol{Y}=\boldsymbol{H} \boldsymbol{P}^{T}+\boldsymbol{N}
$$

where $\boldsymbol{Y} \in \mathbb{C}^{n_{R} \times B}$ is the received sequence, $\boldsymbol{H} \in \mathbb{C}^{n_{R} \times n_{T}}$ is the MIMO channel when $\boldsymbol{H}(i, j)$ denotes the MIMO channel gain between $i^{t h}$ transmitter and $j^{t h}$ receiver and $\boldsymbol{N} \in$ $\mathbb{C}^{n_{R} \times B}$ is the noise matrix. We assume Gaussian noise i.e. $\operatorname{vec}(\boldsymbol{N}) \sim \mathcal{C N}(\mathbf{0}, \boldsymbol{M})$ where $\boldsymbol{M} \in \mathbb{C}^{B n_{R} \times B n_{R}}$ denotes noise covariance matrix. We also assume $\operatorname{vec}(\boldsymbol{H}) \sim \mathcal{C N}(\mathbf{0}, \boldsymbol{R})$ where $\boldsymbol{R} \in \mathbb{C}^{n_{T} n_{R} \times n_{T} n_{R}}$ denotes channel covariance matrix.

\section{Pilot Sequence Design}

In this section, we design the pilot coefficients gathered in the matrix $\boldsymbol{P}$, in order to produce an accurate estimate of the channel $\boldsymbol{H}$-while simultaneously satisfying a set of radar performance criteria. For an accurate channel estimation, one may resort to a minimization of the channel mean-squared error (MSE), expressed as [30], [31]

$$
M S E=\operatorname{tr}\left[\left(\boldsymbol{R}^{-1}+\left(\boldsymbol{P} \otimes \boldsymbol{I}_{n_{R}}\right)^{H} \boldsymbol{M}^{-1}\left(\boldsymbol{P} \otimes \boldsymbol{I}_{n_{R}}\right)\right)^{-1}\right] .
$$

Let $\widetilde{\boldsymbol{P}} \triangleq \mathbf{P} \otimes \mathbf{I}_{n_{R}} \in \mathbb{C}^{B n_{R} \times n_{T} n_{R}}$, and note that using the matrix inversion lemma we have

$$
\begin{aligned}
\boldsymbol{\theta} & \triangleq\left(\boldsymbol{R}^{-1}+\widetilde{\boldsymbol{P}}^{H} \boldsymbol{M}^{-1} \widetilde{\boldsymbol{P}}\right)^{-1} \\
& =\boldsymbol{R}-\boldsymbol{R} \widetilde{\boldsymbol{P}}^{H}\left(\boldsymbol{M}+\widetilde{\boldsymbol{P}} \boldsymbol{R} \widetilde{\boldsymbol{P}}^{H}\right)^{-1} \widetilde{\boldsymbol{P}} \boldsymbol{R}
\end{aligned}
$$

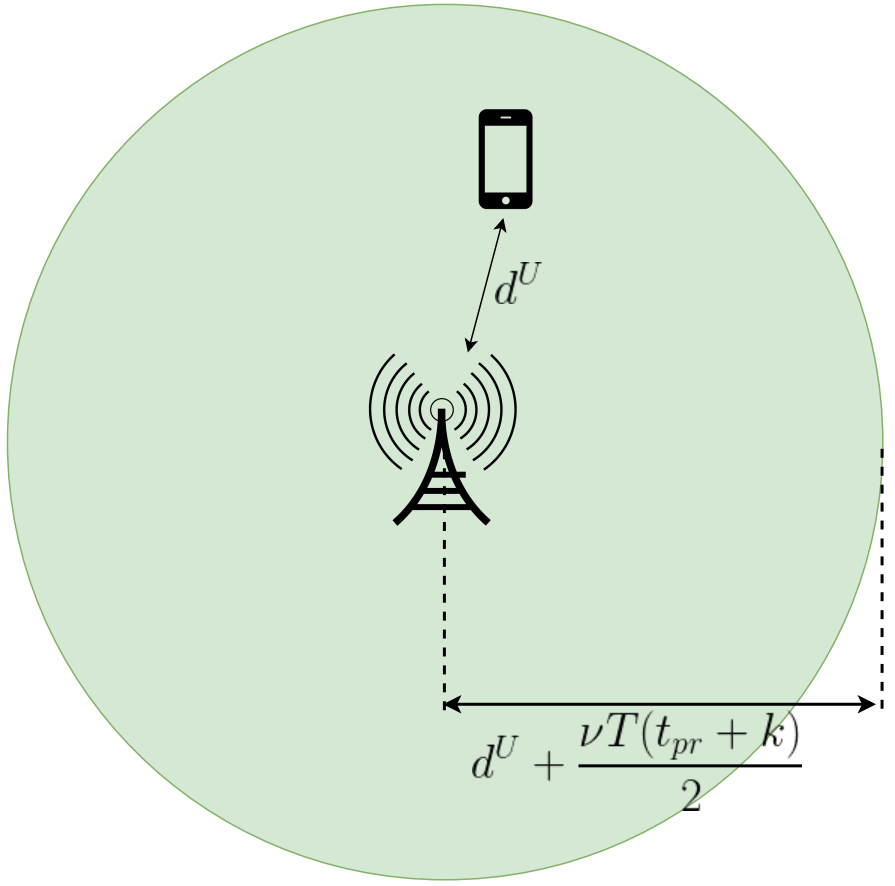

Fig. 2. The radar operational range of ComSens.

where $M S E=\operatorname{tr}[\theta]$. Now let

$$
\begin{gathered}
\boldsymbol{Q} \triangleq\left(\begin{array}{cc}
\boldsymbol{R} & \boldsymbol{R} \widetilde{\boldsymbol{P}}^{H} \\
\widetilde{\boldsymbol{P}} \boldsymbol{R} & \boldsymbol{M}+\widetilde{\boldsymbol{P}} \boldsymbol{R} \widetilde{\boldsymbol{P}}^{H}
\end{array}\right) \in \mathbb{C}^{\left(B+n_{T}\right) n_{R} \times\left(B+n_{T}\right) n_{R}}, \\
\boldsymbol{U} \triangleq\left(\begin{array}{cc}
\boldsymbol{I}_{n_{T} n_{R}} & \left.\mathbf{0}_{n_{T} n_{R} \times B n_{R}}\right)^{T} \in \mathbb{C}^{\left(B+n_{T}\right) n_{R} \times n_{T} n_{R}}
\end{array}\right.
\end{gathered}
$$

and observe that [32],

$$
\boldsymbol{U}^{H} \boldsymbol{Q}^{-1} \boldsymbol{U}=\boldsymbol{\theta}^{-1} .
$$

In light of the above, the authors in [31] propose a cyclic optimization approach to minimizing the MSE in (5): Consider an auxiliary variable $\boldsymbol{V} \in \mathbb{C}^{n_{T} n_{R} \times B n_{R}}$ such that

$$
F(\boldsymbol{V}, \boldsymbol{P}):=\operatorname{tr}\left[\boldsymbol{V}^{H} \boldsymbol{Q} \boldsymbol{V}\right] .
$$

The minimizer $\boldsymbol{V}$ of (11) can be obtained as [33, p. 354]

$$
\boldsymbol{V}_{*}=\left(\begin{array}{c}
\boldsymbol{I}_{n_{T} n_{R}} \\
-\left(\boldsymbol{M}+\widetilde{\boldsymbol{P}} \boldsymbol{R} \widetilde{\boldsymbol{P}}^{H}\right)^{-1} \widetilde{\boldsymbol{P} R}
\end{array}\right)
$$

By substituting (12) in (11), one can verify that

$$
F\left(\boldsymbol{V}_{*}, \boldsymbol{P}\right)=\operatorname{tr}[\boldsymbol{\theta}]=M S E .
$$

Therefore, in order to optimize the MSE we can use a cyclic optimization of (11) with respect to $\boldsymbol{V}$ and $\boldsymbol{P}$. In particular, it was shown in [31] that the optimization of 111 with respect to $\boldsymbol{P}$ can be cast at each (cyclic) iteration as:

$$
\min _{\boldsymbol{P}^{h+1} \in \Omega}\left\|\boldsymbol{P}^{(h+1)}-\boldsymbol{P}_{\Sigma}^{(h)}\right\|_{2}^{2},
$$


where $\boldsymbol{P}_{\Sigma}^{(h)}$ is constructed from $\boldsymbol{P}^{(h)}$ at each iteration (see [31] for details). For the two-part pilot employed in ComSens, define:

$$
\begin{aligned}
& \boldsymbol{P}_{D L}:=\boldsymbol{X} \\
& \boldsymbol{P}_{U L}:=\boldsymbol{Y}
\end{aligned}
$$

where $\boldsymbol{X} \in \mathbb{C}^{B \times n_{T}}$ is the downlink pilot contributing at both radar and communication modes and $\boldsymbol{Y} \in \mathbb{C}^{B \times n_{R}}$ is the uplink pilot which contributes only in communication mode. Thus, (14) becomes

$$
\min _{\boldsymbol{X}, \boldsymbol{Y} \in \Omega}\left\|\boldsymbol{X}-\boldsymbol{X}_{\Sigma}\right\|_{2}^{2}+\left\|\boldsymbol{Y}-\boldsymbol{Y}_{\Sigma}\right\|_{2}^{2}
$$

where the constraint set $\Omega$ is yet to be defined. As indicated earlier, $\boldsymbol{X}$ and $\boldsymbol{Y}$ should have low correlation with each other and $\boldsymbol{X}$ should have an impulse-like autocorrelation. We describe the pilot constraints in three categories:

1) Both pilot sequences should have fixed transmit powers given by

$$
\begin{aligned}
&\left\|\boldsymbol{x}_{q}\right\|_{2}^{2} \leq p, 1 \leq q \leq n_{T} \\
&\left\|\boldsymbol{y}_{l}\right\|_{2}^{2} \leq p, \quad 1 \leq l \leq n_{R}
\end{aligned}
$$

where $\boldsymbol{x}_{q}$ and $\boldsymbol{y}_{l}$ are column vectors of $\boldsymbol{X}$ and $\boldsymbol{Y}$ and $p$ is the power upper-bound.

2) To resolve ambiguity between radar reflections and communication signals, pilot sequences (and their time lags up to $k$ lags) should be uncorrelated to each other; i.e their cross correlation must be zero or very small at least for a number of time lags (forming a zero correlation zone [34], [35]):

$$
\boldsymbol{X}^{T} \boldsymbol{J}_{i} \boldsymbol{Y} \simeq \mathbf{0}^{n_{T} \times n_{R}}, \quad 0 \leq i \leq k,
$$

where $\boldsymbol{J}_{k} \in C^{B \times B}$ is a shift matrix that shifts a matrix by $k$ time lags. Clearly $\boldsymbol{J}_{0}$ is identity matrix.

3) Radar pilot sequence should be impulse-like; i.e. its auto-correlation must be zero or very small at least for a number of time lags:

$$
\boldsymbol{X}^{T} \boldsymbol{J}_{i} \boldsymbol{X} \simeq \mathbf{0}^{n_{T} \times n_{T}}, \quad 0 \leq i \leq k .
$$

Consequently, one can solve the following optimization problem to design our pilot sequences:

$$
\begin{array}{cl}
\min _{\boldsymbol{X}, \boldsymbol{Y} \in \Omega} & \left\|\boldsymbol{X}-\boldsymbol{X}_{\Sigma}\right\|_{2}^{2}+\left\|\boldsymbol{Y}-\boldsymbol{Y}_{\Sigma}\right\|_{2}^{2} \\
\text { s.t. } & \left\|\boldsymbol{x}_{q}\right\|_{2}^{2} \leq p, \quad 1 \leq q \leq n_{T} ; \\
& \left\|\boldsymbol{y}_{l}\right\|_{2}^{2} \leq p, \quad 1 \leq l \leq n_{R} ; \\
& \boldsymbol{x}_{q}^{T} \boldsymbol{J}_{i} \boldsymbol{y}_{l} \leq \epsilon, \quad 1 \leq i \leq k ; \\
& \boldsymbol{x}_{q}^{T} \boldsymbol{J}_{i} \boldsymbol{x}_{q} \leq \epsilon, \quad 1 \leq i \leq k ;
\end{array}
$$

where $\epsilon$ is a very small number (in this paper we use $10^{-5}$ ) to achieve equality constraints. In order to tackle (22) we can use cyclic optimization[36]. We define:

$$
G(\boldsymbol{X}, \boldsymbol{Y}):=\left\|\boldsymbol{X}-\boldsymbol{X}_{\Sigma}\right\|_{2}^{2}+\left\|\boldsymbol{Y}-\boldsymbol{Y}_{\Sigma}\right\|_{2}^{2}
$$

Then one can perform a cyclic procedure to minimize $G(\boldsymbol{X}, \boldsymbol{Y})$ as follows: We start with an initial value $\boldsymbol{Y}=\boldsymbol{Y}^{0}$. Then we comupte $\boldsymbol{X}^{i}$ by tackling minimization problem in Eq. 24 and $\boldsymbol{Y}^{i}$ by tackling minimization problem in Eq. 25). More precisely:

$$
\begin{array}{ll}
\boldsymbol{X}^{i}= & \arg \min _{\boldsymbol{X}} G\left(\boldsymbol{X}, \boldsymbol{Y}^{i-1}\right) \\
\text { s.t. } \quad & \left\|\boldsymbol{x}_{q}\right\|_{2}^{2} \leq p, \quad 1 \leq q \leq n_{T} ; \\
& \boldsymbol{x}_{q}^{T} \boldsymbol{J}_{m} \boldsymbol{y}_{l}^{i-1}=0, \quad 1 \leq m \leq k ; \\
& \boldsymbol{x}_{q}^{T} \boldsymbol{J}_{m} \boldsymbol{x}_{q} \leq \epsilon, \quad 1 \leq m \leq k ; \\
\boldsymbol{Y}^{i}= & \arg \min _{\boldsymbol{Y}} G\left(\boldsymbol{X}^{i}, \boldsymbol{Y}\right) \\
\text { s.t. } \quad & \left\|\boldsymbol{y}_{l}\right\|_{2}^{2} \leq p, \quad 1 \leq l \leq n_{T} ; \\
& \left(\boldsymbol{x}_{q}^{i}\right)^{T} \boldsymbol{J}_{m} \boldsymbol{y}_{l} \leq 0, \quad 1 \leq m \leq k ;
\end{array}
$$

where $1 \leq q \leq n_{T}$ and $1 \leq l \leq n_{R}$. Note that since now the second constraint in both 24) and 25) are affine constraints, we replaced them with equality. Eq. (25) is now a convex optimization problem and solvable using convex optimization. However, the third constraint in 24) is not convex. We can rewrite Eq. 24] in form:

$$
\begin{array}{lll}
\boldsymbol{X}^{i}= & \arg \min _{\boldsymbol{X}} G\left(\boldsymbol{X}, \boldsymbol{Y}^{i-1}\right) & \\
\text { s.t. } & \left\|\boldsymbol{x}_{q}\right\|_{2}^{2} \leq p, & 1 \leq q \leq n_{T} ; \\
& \boldsymbol{x}_{q}^{T} \boldsymbol{J}_{m} \boldsymbol{y}_{l}^{i-1}=0, & 1 \leq m \leq k ; \\
& \boldsymbol{x}_{q}^{T}\left(\boldsymbol{J}_{m}^{T}+\boldsymbol{J}_{m}+2 \boldsymbol{I}_{m}\right) \boldsymbol{x}_{q} \leq 2 p, & 1 \leq m \leq k ;
\end{array}
$$

the third constraint in 26 is now in quadratic convex form since $\left(\boldsymbol{J}_{m}^{T}+\boldsymbol{J}_{m}+2 \boldsymbol{I}_{m}\right)$ is a symmetric positive semi-definite matrix. Note that the optimization problem is still the same (since $\boldsymbol{x}_{q}^{T} \boldsymbol{J}_{m} \boldsymbol{x}_{q} \leq \epsilon$ and $\boldsymbol{J}_{m}^{T}=\boldsymbol{J}_{-m}$ then $\boldsymbol{x}_{q}^{T} \boldsymbol{J}_{m}^{T} \boldsymbol{x}_{q} \leq \epsilon$ also holds and from the first constraint $\boldsymbol{x}_{q}^{T} \boldsymbol{I}_{m} \boldsymbol{x}_{q} \leq p$ ). Now we can follow the steps of the algorithm below to design the pilot sequence.

$\overline{\text { Algorithm } 1 \text { Cyclic Algorithm For Constrained Pilot Sequence }}$ Design

Step 0: Initialize $\boldsymbol{P}_{D L}$ and $\boldsymbol{P}_{U L}$ using a random matrix in $\Omega$. Step 1: Compute the minimizer $V$ of (11) using (12).

Step 2: Update the current design of $\boldsymbol{X}$ and $\boldsymbol{Y}$ by solving cyclic optimization problem (26) and 25) $\mu$ times (or until convergence).

Step 3: Repeat steps 1 and 2 until a stop criterion is satisfied, e.g. $\left|M S E^{(m+1)}-M S E^{(m)}\right|<\eta$ for some given $\eta>0$, where $m$ denotes the outer loop iteration.

\section{Simulation Results}

In this section, we evaluate the performance of the communication method with respect to the channel MSE metric and for the radar mode we illustrate the cross and auto-correlation between two pilot sequences. 


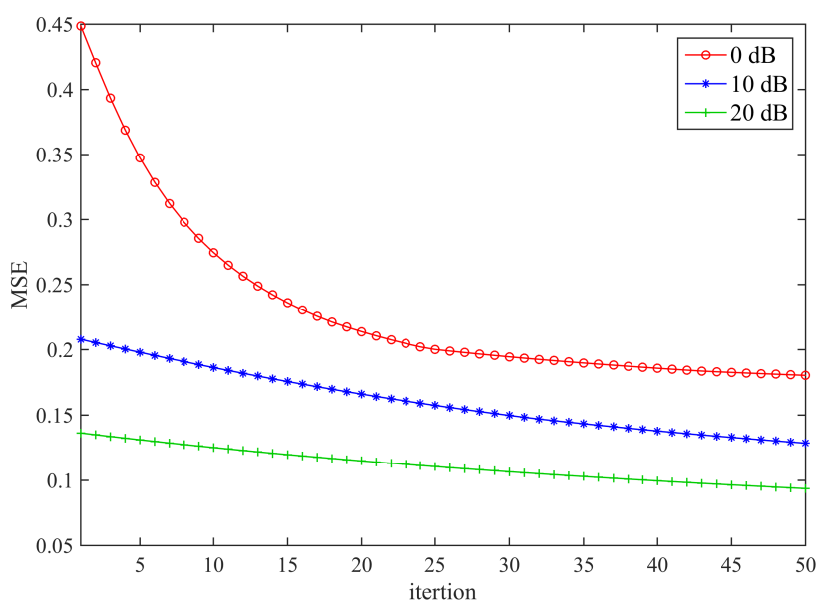

Fig. 3. MSE comparison of different schemes for a $4 \times 4$ MIMO channel where $B=8$. In order to demonstrate the improvement of MSE values through the iterations, values of MSE at each iterations is shown.

\section{A. Simulation Settings}

We used the exponential model to generate covariance matrices. This model is particularly appropriate whenever a control over correlation is required. For a covariance matrix $C$, we let $[\boldsymbol{C}]_{k, l}=\rho^{l-k}$ for $k \leq l$, and $[\boldsymbol{C}]_{k, l}=[\boldsymbol{C}]_{l, k}^{*}$ for $l<k$, with $|\rho|<1$ denoting the correlation coefficient. Furthermore, we assume that both the channel matrix $\mathbf{R}$ and the noise matrix $\mathbf{M}$ follow the Kronecker model; i.e for covariance matrix $\mathbf{R}$ defined as $\mathbf{R}=\left(\mathbf{R}_{\mathbf{T}}^{T} \otimes \mathbf{R}_{\mathbf{R}}\right)$ we suppose $\rho_{r t}=0.9 e^{-j \theta_{r t}}$ and $\rho_{r r}=$ $0.65 e^{-j \theta_{r r}}$ to construct $\mathbf{R}_{\mathbf{T}}$ and $\mathbf{R}_{\mathbf{R}}$ (at the transmit side and the receive side, respectively) using exponential model. Also, for covariance matrix of noise $\mathbf{M}$ defined as $\mathbf{M}=\left(\mathbf{M}_{\mathbf{T}}^{T} \otimes \mathbf{M}_{\mathbf{R}}\right)$ where $\mathbf{M}_{\mathbf{R}}=\mathbf{R}_{\mathbf{R}}$, we let $\rho_{m t}=0.8 e^{-j \theta_{m t}}$ to construct $\mathbf{M}_{\mathbf{T}}$ at the transmitter side.The phase arguments $\left(\theta_{r t}, \theta_{r r}, \theta_{m t}\right)$ appearing above were chosen randomly as $(0.8349 \pi, 0.4289 \pi, 0.5361 \pi)$.

We also normalize $\mathbf{R}$ and $\mathbf{M}$ such that $\operatorname{tr}\{\mathbf{R}\}=1$ and $\operatorname{tr}\{\mathbf{M}\}=1$, and define the pilot sequence-to-noise ratio (SNR) as SNR $\triangleq \gamma$, and $\gamma=\|\boldsymbol{P}\|_{F}^{2}$ denotes the total training energy. We consider $\gamma=B n_{T}$, and set the stop threshold of the iteration loop in Algorithm I as $\eta=10^{-5}$.

\section{B. Channel MSE Metric}

We show the performance of the suggested approach for communication purposes using MSE as the figure of merit. We consider a $4 \times 4$ MIMO channel with $B=8$. The results are shown in Fig. 3 . For each power, we have used the proposed method 50 times, using different initializations, and have reported the average of the obtained MSE values. It can be observed from Fig. 3 that the proposed method performs better in each iteration until it converges to the optimal MSE.

\section{Radar Pilot Sequence Specifications}

To ensure radar part of the system performs properly, our pilot sequence for radar part should have very small auto-correlation for at least a range of time lags so that this pilot sequence have an

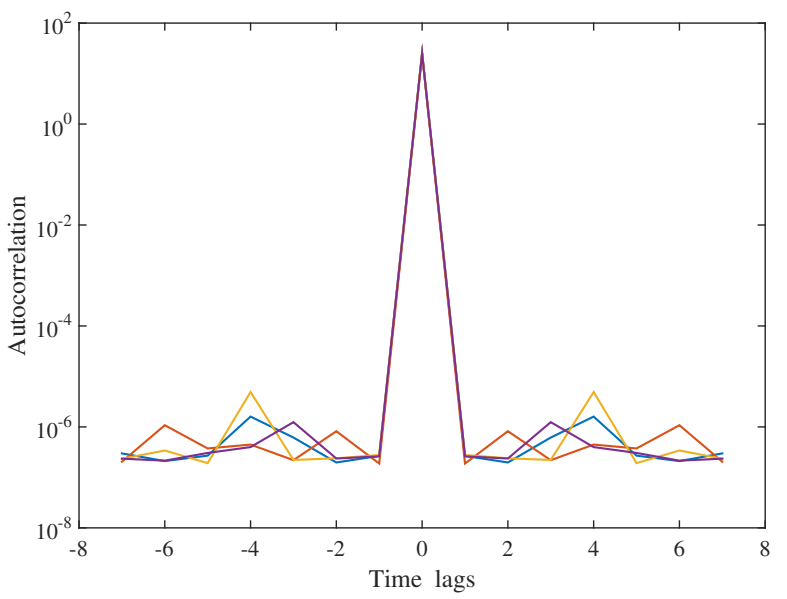

Fig. 4. Autocorrelation of radar pilot signal $\left(\boldsymbol{x}_{l}^{T} \boldsymbol{J}_{i} \boldsymbol{x}_{l}\right)$ where $1 \leq l \leq 8$, and each $l$ denotes a transmit antenna so we have totally 4 autocorrelation plots in this figure, and also $-8 \leq i \leq 8$ denote time lags

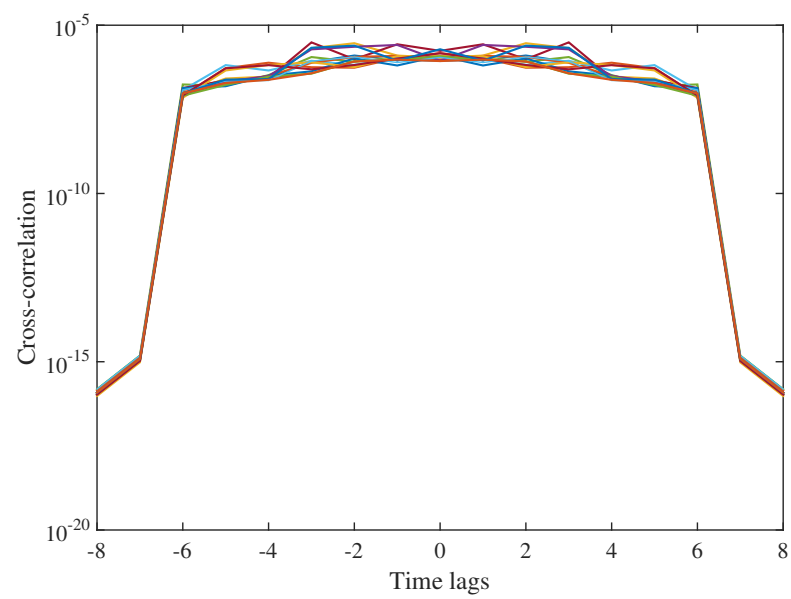

Fig. 5. Cross-correlation of radar and communication pilot signals $\left(\boldsymbol{x}_{q}^{T} \boldsymbol{J}_{i} \boldsymbol{y}_{l}\right)$ where $1 \leq q \leq 8$, and each $q$ denotes different transmit antenna, $1 \leq l \leq 8$, and each $l$ denotes different receive antenna so we have totally 16 cross-correlation plots in this figure, and also $-8 \leq i \leq 8$ denote time lags

impulse like shape. In Fig. 4 this auto-correlation is shown. For each lag, auto-correlation level is shown in $\mathrm{dB}$. Fig. 4 shows that auto-correlation levels for time lags 2-8 are almost zero compared to autocorrelation for the first lag. Which gives us the impulselike correlation for the pilot sequence contributing in sensing mode.

\section{Correlation of pilots}

The key factor for our system to distinguish between radar signal and communication signal is that two pilot sequences should be uncorrelated with each other for a number of time lags. Fig. 5 shows cross-correlation between two pilot signals for our simulations in $\mathrm{dB}$. As it is obvious from simulations results, correlation between these two signals are really small so they can be assumed uncorrelated. 


\section{CONCLUSION}

The idea of designing pilot sequences for a communication system to be able to operate also in an integrated radar mode has been proposed and the protocol and limitations has been explained. We evaluate the channel MSE for communication system and show that pilot sequences are uncorrelated, and also one of the sequences have impulse-like correlation (suitable for radar sensing). The proposed system can perform as a radar and communication system. Considering the communication operation as the primary also lays the ground for making the radar systems ubiquitous.

\section{REFERENCES}

[1] A. Sani and A. Vosoughi, "Bandwidth and power constrained distributed vector estimation in wireless sensor networks," in Military Communications Conference, MILCOM 2015-2015 IEEE. IEEE, 2015, pp. 1164-1169.

[2] S. Hajizadeh and G. A. Hodtani, "Three-receiver broadcast channels with side information," in Information Theory Proceedings (ISIT), 2012 IEEE International Symposium on. IEEE, 2012, pp. 393-397.

[3] T. X. Tran, M.-P. Hosseini, and D. Pompili, "Mobile edge computing: Recent efforts and five key research directions," MMTC CommunicationsFrontiers, vol. 12, no. 4, pp. 29-34, 2017.

[4] M. Karimzadeh Kiskani, B. Azimdoost, and H. Sadjadpour, "Effect of social groups on the capacity of wireless networks," IEEE Transactions on Wireless Communications, vol. 15, no. 1, pp. 3-13, 2016.

[5] M.-P. Hosseini, M. R. Nazem-Zadeh, D. Pompili, K. Jafari, K. Elisevich, and H. Soltanian-Zadeh, "Support vector machine with nonlinear-kernel optimization for lateralization of epileptogenic hippocampus in mr images," in Engineering in Medicine and Biology Society (EMBC), 2014 36th Annual International Conference of the IEEE. IEEE, 2014, pp. 1047-1050.

[6] V. Naghshin, M. C. Reed, and N. Aboutorab, "Coverage analysis of packet multi-tier networks with asynchronous slots," IEEE Transactions on Communications, vol. 65, no. 1, pp. 200-215, Jan 2017.

[7] A. Sani and A. Vosoughi, "Distributed vector estimation for power-and bandwidth-constrained wireless sensor networks," IEEE Transactions on Signal Processing, vol. 64, no. 15, pp. 3879-3894, 2016.

[8] S. Vahidian, S. Assa, and S. Hatamnia, "Relay selection for securityconstrained cooperative communication in the presence of eavesdropper's overhearing and interference," IEEE Wireless Communications Letters, vol. 4, no. 6, pp. 577-580, Dec 2015.

[9] M.-P. Hosseini, T. Tran, D. Pompili, K. Elisevich, and H. Soltanian-Zadeh, "Deep learning with edge computing for localization of epileptogenicity using multimodal rs-fmri and eeg big data," in IEEE International Conference on Autonomic Computing (ICAC). IEEE, 2017.

[10] M. Yazdani, J. K. Lee, J. Mautz, E. Arvas, and K. Sun, "Shortcut waves in a radially uniaxial sphere," in 2015 IEEE International Symposium on Antennas and Propagation USNC/URSI National Radio Science Meeting, July 2015, pp. 1112-1113.

[11] V. Naghshin, A. M. Rabiei, N. C. Beaulieu, and B. Maham, "Accurate statistical analysis of a single interference in random networks with uniformly distributed nodes," IEEE Communications Letters, vol. 18, no. 2, pp. 197-200, February 2014.

[12] M. K. Kiskani and H. Sadjadpour, "Throughput analysis of decentralized coded content caching in cellular networks," IEEE Transactions on Wireless Communications, vol. 16, no. 1, pp. 663-672, 2017.

[13] M. H. Moghaddam, M. J. Azizipour, S. Vahidian, and B. Smida, "A framework for super-resolution of scalable video via sparse reconstruction of residual frames," arXiv preprint arXiv:1707.09926, 2017.

[14] M. Yazdani, J. Mautz, L. Murphy, and E. Arvas, "High-frequency scattering from radially uniaxial dielectric sphere," IEEE Antennas and Wireless Propagation Letters, vol. 14, pp. 1577-1581, 2015.

[15] A. Hassanien, M. G. Amin, Y. D. Zhang, and F. Ahmad, "A dual function radar-communications system using sidelobe control and waveform diversity," in 2015 IEEE Radar Conference (RadarCon), May 2015, pp. $1260-1263$.

[16] S. D. Blunt, P. Yatham, and J. Stiles, "Intrapulse radar-embedded communications," IEEE Transactions on Aerospace and Electronic Systems, vol. 46, no. 3, pp. 1185-1200, July 2010.

[17] J. Euzire, R. Guinvarc'h, M. Lesturgie, B. Uguen, and R. Gillard, "Dual function radar communication time-modulated array," in 2014 International Radar Conference, Oct 2014, pp. 1-4.
[18] Y. L. Sit, C. Sturm, L. Reichardt, T. Zwick, and W. Wiesbeck, "The OFDM joint radar-communication system: An overview," in Proc. Int. Conf. Advances in Satellite and Space Communications (SPACOMM 2011), 2011, pp. 69-74.

[19] C. Sturm, T. Zwick, and W. Wiesbeck, "An OFDM system concept for joint radar and communications operations," in Vehicular Technology Conference, 2009. VTC Spring 2009. IEEE 69th, April 2009, pp. 1-5.

[20] Y. L. Sit, L. Reichardt, C. Sturm, and T. Zwick, "Extension of the OFDM joint radar-communication system for a multipath, multiuser scenario," in 2011 IEEE RadarCon (RADAR), May 2011, pp. 718-723.

[21] C. Sturm and W. Wiesbeck, "Waveform design and signal processing aspects for fusion of wireless communications and radar sensing," Proceedings of the IEEE, vol. 99, no. 7, pp. 1236-1259, July 2011.

[22] M. Mousaei and B. Smida, "Optimizing pilot overhead for ultra-reliable short-packet transmission," in 2017 IEEE International Conference on Communications (ICC), May 2017, pp. 1-5.

[23] B. Hassibi and B. M. Hochwald, "How much training is needed in multipleantenna wireless links?" IEEE Transactions on Information Theory, vol. 49 no. 4, pp. 951-963, 2003

[24] S. Hatamnia, S. Vahidian, S. Aïssa, B. Champagne, and M. AhmadianAttari, "Network-coded two-way relaying in spectrum-sharing systems with quality-of-service requirements," IEEE Transactions on Vehicular Technology, vol. 66, no. 2, pp. 1299-1312, 2017.

[25] M. Karimzadeh Kiskani and H. R. Sadjadpour, "Capacity of cellular networks with femtocache," in Computer Communications Workshops (INFOCOM WKSHPS), 2016 IEEE Conference on. IEEE, 2016.

[26] S. Vahidian, E. Soleimani-Nasab, S. Assa, and M. Ahmadian-Attari, "Bidirectional af relaying with underlay spectrum sharing in cognitive radio networks," IEEE Transactions on Vehicular Technology, vol. 66, no. 3, pp. 2367-2381, March 2017.

27] M. K. Kiskani and H. R. Sadjadpour, "Multihop caching-aided coded multicasting for the next generation of cellular networks," IEEE Transactions on Vehicular Technology, vol. 66, no. 3, pp. 2576-2585, 2017.

[28] V. Naghshin and M. C. Reed, "On capacity and association area characterization in small cell-based multi-tier networks," IEEE Wireless Communications Letters, vol. 4, no. 5, pp. 505-508, Oct 2015.

[29] S. Hataminia, S. Vahidian, M. Mohammadi, and M. Ahmadian-Attari, "Performance analysis of two-way decode-and-forward relaying in the presence of co-channel interferences," IET Communications, vol. 8, no. 18 pp. 3349-3356, 2014.

[30] N. Shariati, J. Wang, and M. Bengtsson, "Robust training sequence design for correlated MIMO channel estimation," IEEE Transactions on Signal Processing, vol. 62, no. 1, pp. 107-120, Jan. 2014.

[31] M. Soltanalian, M. M. Naghsh, N. Shariati, P. Stoica, and B. Hassibi, "Training signal design for correlated massive mimo channel estimation," IEEE Transactions on Wireless Communications, vol. 16, no. 2, pp. 11351143, Feb 2017.

[32] K. B. Petersen, M. S. Pedersen et al., The matrix cookbook. Technical University of Denmark, 2008.

[33] P. Stoica and R. Moses, Spectral Analysis of Signals. New Jersey: Prentice Hall, 2005. [Online]. Available: http://user.it.uu.se/ ps/ref.html

[34] P. Z. Fan, N. Suehiro, N. Kuroyanagi, and X. M. Deng, "Class of binary sequences with zero correlation zone," Electronics Letters, vol. 35, no. 10, pp. 777-779, May 1999.

[35] H. Torii, M. Nakamura, and N. Suehiro, "A new class of zero-correlation zone sequences," IEEE Transactions on Information Theory, vol. 50, no. 3, pp. 559-565, March 2004.

[36] P. Stoica and Y. Selen, "Cyclic minimizers, majorization techniques, and the expectation-maximization algorithm: a refresher," IEEE Signal Processing Magazine, vol. 21, no. 1, pp. 112-114, Jan 2004. 